\title{
An environmentally friendly method to improve the quality of recycled concrete aggregates
}

\author{
Liang Wang ${ }^{\mathrm{a}}$, Jialai Wang ${ }^{\mathrm{b},}$, Xin Qian ${ }^{\mathrm{b}}$, Peiyuan Chen ${ }^{\mathrm{b}}$, Ying Xu ${ }^{\mathrm{a}}$, Jinxing Guo $^{\mathrm{a}}$ \\ ${ }^{a}$ School of Civil Engineering and Architecture, Anhui University of Science and Technology, \\ Huainan 232001, Anhui, P.R. China \\ ${ }^{\mathrm{b}}$ Department of Civil, Construction, and Environmental Engineering, The University of \\ Alabama, Tuscaloosa, AL 35487, USA
}

\begin{abstract}
Recycled Concrete Aggregates (RCAs) have inferior qualities compared with natural aggregates, mainly attributed to the porous nature of the attached cement mortar. To improve the quality of the RCAs, an environmentally friendly and cost-effective method is proposed to treat RCAs in this study. In this method, RCAs are first soaked in acetic acid solution, in which the acetic acid reacts with cement hydration products attached to the surface of the RCAs. This reaction weakens the attached mortar, making it possible to remove it from the RCAs by mechanical rubbing later. The treated RCAs have lower water absorption and less cement mortar attached. Once used as aggregates in new concrete, these RCAs can enhance the compressive strength of the concrete at 28 days up to $25 \%$. It is safe and clean to apply this new method since no dangerous chemical is used and no detrimental chemicals are introduced into the treated RCAs. More importantly, zero hazardous waste solution is
\end{abstract}

\footnotetext{
* Corresponding author. Tel.: +1 205348 6786; fax: +1 2053480783.

Email address: jwang@eng.ua.edu (J.Wang) 
generated since the waste solution of the treatment can be used to produce high value-added products, permanently store $\mathrm{CO}_{2}$, and regenerate acetic acid. As an example, the waste solution of the treatment was used as admixture for new concrete, which improved the strength of the concrete by $14 \%$.

Keywords: recycled concrete aggregate; water absorption; acetic acid; mechanical rubbing; concrete; microstructure; waste solution.

\section{Introduction}

Concrete, as the most widely used construction materials, creates two major negative impacts on environment. On one hand, manufacturing of concrete consumes large amounts of natural resources. For example, current concrete industry demands more than 20 billion tons of raw materials (coarse aggregate) every year, and this demand will be doubled in the next 20 to 30 years [1-2]. On the other hand, all produced concrete will be demolished sooner or later, creating a large amount of Construction and Demolition (C\&D) waste, especially concrete waste. Actually, C\&D waste has already become one of the largest waste flows in the world. An estimated $900 \mathrm{Mt}$ of C\&D wastes are produced every year in Europe, the US and Japan [3]. In the mainland of China, more than $200 \mathrm{Mt}$ of waste concrete are currently produced every year [4].

A promising method to mitigate these two negative environmental impacts is recycling $C \& D$ wastes to produce alternative aggregates for concretes. However, Recycled Concrete Aggregate (RCAs) are mainly limited to low-value added applications such as roadbed materials because of 
their inferior qualities compared with Natural Aggregates (NAs). This is because a layer of residual cement mortar is coated on the surface of the NAs in the RCAs. The porous nature of this residual mortar introduces undesirable properties to the RCAs, including higher water absorption, lower strength, and higher crushing value. Consequently, concrete made from RCAs usually has lower workability and higher creep and shrinkage than those of concrete using NAs $[2,5-7]$.

A viable way to improve the quality of RCAs is to remove the porous residual cement mortar from the NAs. To this end, a few methods have been developed, including thermal process [8-12] and chemical process [13-14]. In thermal process [8], RCAs are first heated to a temperature high enough to dehydrate the cement hydration products. This process can significantly weaken the residual mortar so that it can be separated from the NA by mechanical rubbing. However, heating the RCAs not only consumes large amount of thermal energy, but also produce additional carbon dioxide emission. Chemical process uses a low-concentration acid (hydrochloric acid, sulfuric acid, and phosphoric acid), instead of heating, to weaken the cement mortar attached to the RCA, as demonstrated in [13-14]. To this end, RCAs are first soaked in hydrochloric acid or sulfuric acid solution to dissolve some hydration products of the cement. Some loose and cracked mortar can be removed from the RCAs by this treatment, leading to up to $28 \%$ reduction in water absorption of RCAs. The mechanical properties of the concrete produced with the treated RCAs as aggregate can be significantly improved. However, strong acids (hydrochloric acid and sulfuric acid) are used in this method, which not only poses a threat to the safety of the workers, but also adds detrimental ions such as $\mathrm{Cl}^{-}$and $\mathrm{SO}_{4}{ }^{2-}$ to the RCAs. These ions can cause 
durability issues in concrete with these RCAs. Therefore, large amount of water is needed to wash the treated RCAs. However, disposal of this washing water and the residual of the treatment solution will create new environmental problems.

With an aim to overcome the drawbacks of existing chemical process, this study proposes using low concentration acetic acid to treat RCAs so that high quality aggregates can be produced. This new method is illustrated in Fig. 1.RCAs will first be soaked in low concentration acetic acid (1\% to 5\%). Acetic acid can react with calcium hydroxide, calcium carbonate (in carbonated RCAs), and calcium silicate hydrate in the cement mortar attached to the surface of the RCA as described by

$$
\begin{gathered}
\mathrm{CH}+\mathrm{CH}_{3} \mathrm{COOH}_{(\mathrm{l})} \rightarrow \mathrm{Ca}^{2+}{ }_{(\mathrm{aq})}+\mathrm{CH}_{3} \mathrm{COO}^{-}(\mathrm{aq})+\mathrm{H}_{2} \mathrm{O}_{(\mathrm{aq})}, \\
\mathrm{CaCO}_{3}+\mathrm{CH}_{3} \mathrm{COOH}_{(\mathrm{l})} \rightarrow \mathrm{Ca}^{2+}{ }_{(\mathrm{aq})}+\mathrm{CH}_{3} \mathrm{COO}^{-}(\mathrm{aq})+\mathrm{CO}_{2(\mathrm{~g})}, \\
\mathrm{C}-\mathrm{S}-\mathrm{H}+\mathrm{CH}_{3} \mathrm{COOH}_{(\mathrm{l})} \rightarrow \mathrm{Ca}^{2+}{ }_{(\mathrm{aq})}+\mathrm{CH}_{3} \mathrm{COO}^{-}{ }_{(\mathrm{aq})}+\mathrm{H}_{2} \mathrm{O}_{(\mathrm{aq})}+\mathrm{SiO}_{2(\mathrm{~s})},
\end{gathered}
$$

where $\mathrm{CH}$ is calcium hydroxide, $\mathrm{C}-\mathrm{S}-\mathrm{H}$ is calcium silicate hydrate. These reactions can weaken the attached mortar, making it possible to remove most of them from the RCA by mill crushing in the next step, as shown in Fig. 1. This treatment can significantly enhance the quality of produced aggregate since much less mortar is attached to it.

Compared with existing chemical processes, this new method enjoys many advantages:

1) Lower cost: Acetic acid is cheaper than strong acid such as $\mathrm{HCl}$ and $\mathrm{H}_{2} \mathrm{SO}_{4}$. More importantly, acetic acid can be partially regenerated as shown in Fig.1. 
2) Safer: Acetic acid solution is much milder, posing less threat to the safety of the workers. It is also much easier to ship acetic acid than strong acids (hydrochloric acid, sulfuric acid, and phosphoric acid).

3) Cleaner: No detrimental ions are introduced into RCAs. Unlike in the case of using hydrochloric acid or sulfuric acid, no chemical ions such as $\mathrm{Cl}^{-}$and $\mathrm{SO}_{4}{ }^{2-}$ will be introduced into the RCAs. No evidence so far suggests that acetate ions can cause deterioration of concrete [15-17]. Therefore, there is no need to wash the treated aggregates, which will save a large amount of water and the cost of the treatment.

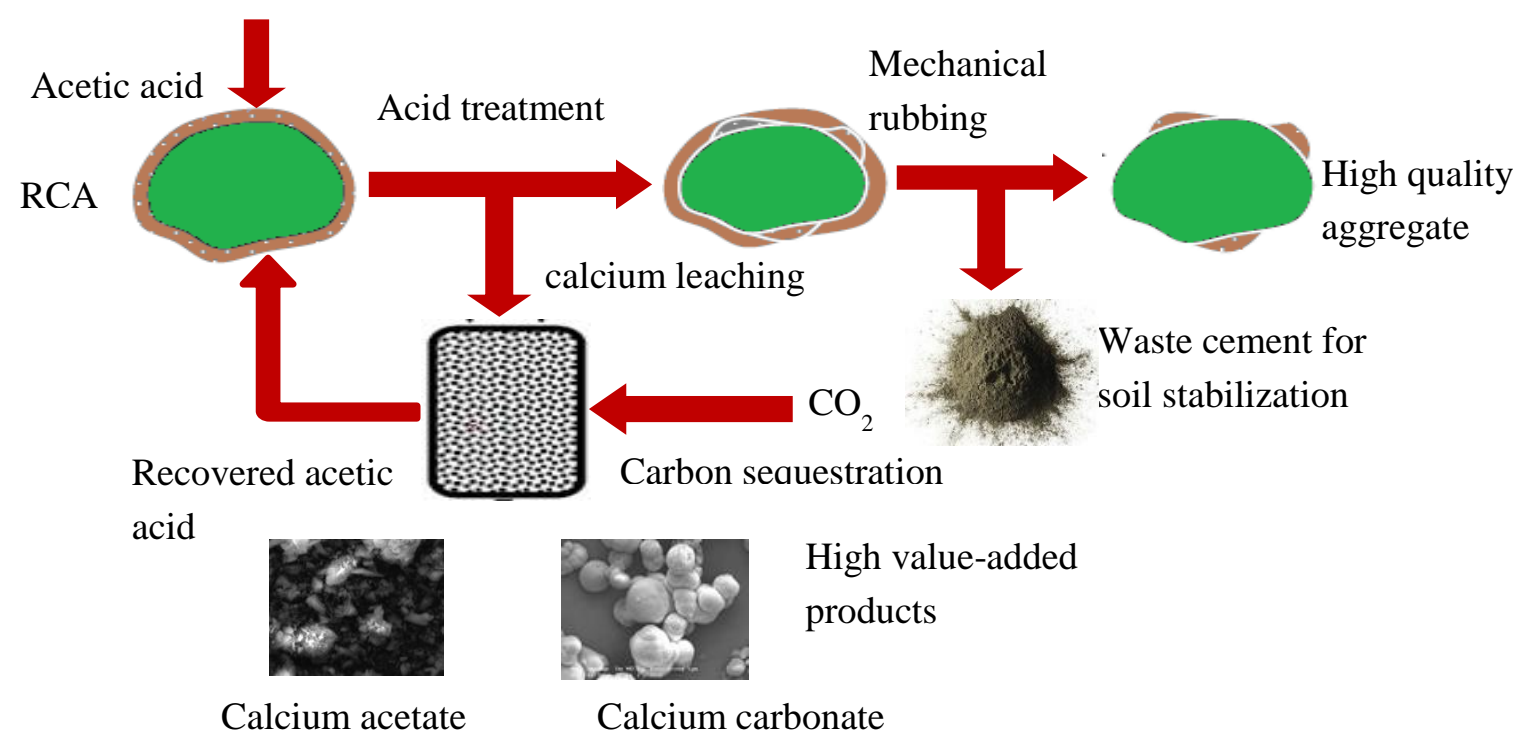

Fig. 1 New approach to treat RCA for value-added products

More importantly, value-added applications or products can be made from the waste solution of the treatment, which is rich in calcium and acetate ions. For example, $\mathrm{CO}_{2}$ can be bubbled into the solution so that some Precipitated Calcium Carbonate (PCC) can be produced, as shown in 
Fig. 1. Through properly controlling the reaction conditions, vaterite, one metastable polymorph of calcium carbonate can be produced [18]. In ambient environment, vaterite has higher solubility and surface free energy than those of other two polymorphs of calcium carbonate (aragonite and calcite). Together with its biocompatibility, colorlessness, low density, and porous structure [19-20], vaterite is a high value-added product which has found many applications in drug delivery, paper making, reaction catalyzing, pollution adsorbing, plastics and rubbers manufacturing. Vaterite can be transformed into calcite without through the intermediate phase aragonite through a dissolution-recrystallization process. In this process, vaterite dissolves releasing calcium and carbonate into solution, which then re-precipitates on the surface of the growing calcite crystals [21]. Due to this phase transformation process, metastable $\mathrm{CaCO}_{3}$ can be used as a binder to cement aggregates together, as demonstrated in recent applications in concrete [22]. In this way, calcium in RCA can be recycled as binder again.

Producing PCC from the waste solution can permanently store $\mathrm{CO}_{2}$ in the produced PCC to further reduce the carbon emission of concrete. In addition, acetic acid can be recovered by $\mathrm{CO}_{2}$ bubbling because carbonic acid is stronger than acetic acid. The rest solution can also be directly used as admixture for concrete, which can enhance the compressive strength of concrete, as demonstrated in this study, or to produce calcium acetate, which is even more expensive than the acetic acid.

The major objective of this paper is to confirm that high quality aggregate can be produced by treating RCAs in low concentration acetic acid and that waste solution of the treatment can be used as chemical admixture to improve the strength of the concrete. Producing other value-added 
products from the waste solution of the treatment and $\mathrm{CO}_{2}$ storage was presented in a companion paper [18].

\section{Materials and methods}

\subsection{Materials}

Ordinary Portland Cement (OPC) was used as the main binder for concrete specimens. Its chemical composition is given in Table 1. The coarse RCA used in this study was generated from crushed waste concrete cubes collected from the Concrete Laboratory at the Center of Construction Quality Supervision and Inspection in Huainan, Anhui, China. The fine aggregate used is natural river sand from Huaihe River in Huainan area with fineness modulus of 2.56.

Table 1. Chemical composition of the OPC used in this study

\begin{tabular}{lllllllll} 
Composition & $\mathrm{SiO}_{3}$ & $\mathrm{Al}_{2} \mathrm{O}_{3}$ & $\mathrm{Fe}_{2} \mathrm{O}_{3}$ & $\mathrm{MgO}$ & $\mathrm{CaO}$ & $\mathrm{SO}_{3}$ & $\mathrm{~K}_{2} \mathrm{O}$ & Others \\
\hline$(\%)$ & 21.3 & 4.87 & 4.92 & 1.21 & 63.21 & 2.03 & 0.69 & 0.82 \\
\hline
\end{tabular}

\subsection{Treating and characterizing the RCA}

Oven-dried RCA samples were first immersed in acetic acid solution at ambient temperature. The concentration of acid solution and the duration of immersion are two key factors affecting the treatment results. Three different acid concentrations $(1 \%, 3 \%, 5 \%)$, and three different immersion durations (1, 3 and 5 days) were used in this study with an aim to obtain the optimal treatment conditions. The change of the $\mathrm{pH}$ value of the acid solution was recorded to monitor the reaction progress between the acetic acid and the cement mortar. After specified duration of 
immersion, RCA samples were taken out and dried in a ventilated oven at $105^{\circ} \mathrm{C} \pm 5^{\circ} \mathrm{C}$ for no less than $24 \mathrm{~h}$ and until two successive weights at intervals of $2 \mathrm{~h}$ show a difference less than $0.2 \mathrm{~g}$. Then, the specimen was sieved using a $5 \mathrm{~mm}$ sieve to separate the fine RCAs from the coarse ones. Only the coarse RCAs were used to make concrete. The water absorption and the apparent density of the coarse RCA sample were then measured.

\subsection{Manufacturing and characterizing concrete specimens with the treated RCA as coarse}

\section{aggregates}

Mix design of concrete was proportioned according to China standard (JGJ 55-2011, GB/T

25177-2010 and JGJ/T240-2011). Constant effective water/cement ratio of 0.46 was used for all concrete mixtures based on the slump, cohesion and water-retaining property of try mixing concrete. Concrete cubic specimens with size of $100 \mathrm{~mm}$ were made using the RCA as the coarse aggregate with mix proportions shown in Table 2 . All specimens were cured in curing room with temperature at $20^{\circ} \mathrm{C} \pm 1^{\circ} \mathrm{C}$ and relative humidity no less than $95 \%$. Four types of RCA were used in the concrete: untreated, treated by acetic acid solution, treated by acetic acid solution and then washed by clean water (noted as "W"), treated by acetic acid and then mechanically rubbed through strong vibration (noted as "M"), and treated by acetic acid, washed by clean water, and mechanically rubbed (noted as "WM"). As a comparison, a group of concrete was also made with natural aggregate, referred to as "NA" in this study.

The compressive strengths of the concrete samples were measured to evaluate the effect of the proposed treatment on the quality of the RCA. Scanning electron microscopy (SEM) and 
X-ray diffraction (XRD) were used to examine the microstructure and minerals of the treated and untreated RCAs. SEM was also used to study the difference of the microstructures of the concrete induced by treating the RCA using the proposed method.

Table 2 Mix design used to make concrete with RCAs

\begin{tabular}{lllll}
\hline Constituents & Water & Cement & Sand & RCA \\
\hline Proportion $\left(\mathrm{kg} / \mathrm{m}^{3}\right)$ & 215 & 467 & 689 & 1029 \\
\hline
\end{tabular}

\subsection{Characterizing the waste solution of the treatment}

The waste solution after treating the RCA was analyzed to determine what were dissolved by the acetic acid through Energy Disperse Spectroscopy (EDS) and XRD analysis. To this end, the waste acetic solution samples were first heated to distill all water. The residual powders were then examined by SEM and EDS to identify chemical elements in the solution. To carry out XRD analysis on the waste solution samples, metal element (magnesium, sulfur, aluminum) in the waste solution were first removed by adding calcium hydroxide which can react with the metal elements to form precipitants. After filtering out these precipitants, the solution was dried slowly to be used for XRD analysis.

\subsection{Evaluating the waste solution of the treatment as an admixture for concrete}

The waste solution of the treatment can be directly added into concrete as a chemical admixture. To evaluate its effect on concrete, two group of concrete samples were cast, one (the control group) without and the other one with the waste solution in the mixing water. The mix 
design of these two groups of concrete samples is given in Table 3. The compressive strength of these two groups were measured and compared.

Table 3 Mix design of the concrete with waste treatment solution as an admixture

\begin{tabular}{lcccc}
\hline Constituents & Water or waste solution & Cement & Sand & RCA \\
\hline Proportion $\left(\mathrm{kg} / \mathrm{m}^{3}\right)$ & 225 & 468 & 717 & 990 \\
\hline
\end{tabular}

\section{Results and discussion}

\subsection{Characterization of the treated RCA}

\subsubsection{Water absorption and apparent density of the treated RCA}

Table 4 shows the water absorption and apparent density of the RCA before and after acetic acid treatment. After acetic treatment, water absorptions of all RCA samples were reduced by $9 \%$ to $19 \%$. Lowest water absorption was achieved by using $1 \%$ acetic acid to treat the RCA. However, further reduction of the water absorption cannot be obtained by using higher concentration of the acetic acid. As shown in Table 4, water absorption of the treated RCA actually increases with the concentration of the acetic acid. This can be attributed to that more hydration products, and possibly some NAs were dissolved by the acetic acid at higher concentration. As a result, more pores were produced in the treated RCA samples, leading to higher water absorption. For acids with concentrations of $1 \%$ and $3 \%$, there is no clear trend showing that 3 or 5-day immersion can lead to lower water absorption of the RCA than 1-day immersion. This suggests that 1-day immersion is sufficient to treat the RCA when these two 
concentrations of acetic acid are used. When 5\% acetic acid is used, longer immersion actually increases the water absorption of the RCA, as shown in Table 4 . This clearly indicates that more hydration products/NAs have been removed by $5 \%$ acetic acid with longer immersion time. Apparent densities of all treated RCA samples are higher than that of the untreated one, confirming that some loose part of the RCAs was removed by the acetic acid treatment.

Figure 2 shows the $\mathrm{pH}$ values of the acetic acid solution changing with the immersion duration. As anticipated, $\mathrm{pH}$ values in all three solutions increase with immersion time due to the reaction between the RCA and the acid. It seems that $\mathrm{pH}$ value of the $1 \%$ acetic acid solution evolves with immersion duration in a different way from that of the other two. Within the first few hours of immersion, the $\mathrm{pH}$ value of the $1 \%$ acetic acid increases much slower, suggesting very slow reaction between the $1 \%$ acetic acid and the RCA at this stage. Very fast reaction between the RCA and 3\% and 5\% acetic acid solutions can be seen from Fig. 2 in the first hour of immersion. Some air bubbles were produced in this stage, which are $\mathrm{CO}_{2}$ and were produced by the reaction between the acetic acid and the carbonated cement paste. This reaction can consume significant amount of acid, leading to fast increase of $\mathrm{pH}$ value in these two acid solutions in the first hour of immersion, as shown in Fig. 2. In the case of using $1 \%$ acetic acid, the concentration of the acid may be not high enough to trigger the fast reaction between the carbonate and the acid. As a result, its $\mathrm{pH}$ value increases at a much lower rate in this stage. The $\mathrm{pH}$ values of all solutions flatten after $24 \mathrm{~h}$ immersion, suggesting that most chemical reactions are completed within $24 \mathrm{~h}$. This indicates that 1 day immersion is sufficient and longer immersion may be unnecessary. This is in agreement with the observation shown in Table 4. For 
this reason, 1 day immersion was used in all tests thereafter in this study.

Table 4 Effects of treatment conditions on properties of the RCA

\begin{tabular}{|c|c|c|c|c|c|c|c|c|c|c|}
\hline \multirow[t]{3}{*}{ Properties of the RCA } & \multirow{3}{*}{$\begin{array}{l}\text { Untreated } \\
\text { RCA }\end{array}$} & \multicolumn{9}{|c|}{ Acid concentrations and duration of immersion (Days) } \\
\hline & & \multicolumn{3}{|c|}{$1 \%$ Acetic acid } & \multicolumn{3}{|c|}{$3 \%$ Acetic acid } & \multicolumn{3}{|c|}{$5 \%$ Acetic acid } \\
\hline & & $1 \mathrm{~d}$ & $3 \mathrm{~d}$ & $5 \mathrm{~d}$ & $1 \mathrm{~d}$ & $3 d$ & $5 d$ & $1 \mathrm{~d}$ & $3 d$ & $5 \mathrm{~d}$ \\
\hline Water absorption (\%) & 5.95 & 4.94 & 4.90 & 4.95 & 5.06 & 5.14 & 4.99 & 5.05 & 5.26 & 5.41 \\
\hline Apparent density $\left(\mathrm{g} / \mathrm{cm}^{3}\right)$ & 2.56 & 2.61 & 2.61 & 2.58 & 2.61 & 2.61 & 2.60 & 2.62 & 2.61 & 2.61 \\
\hline
\end{tabular}

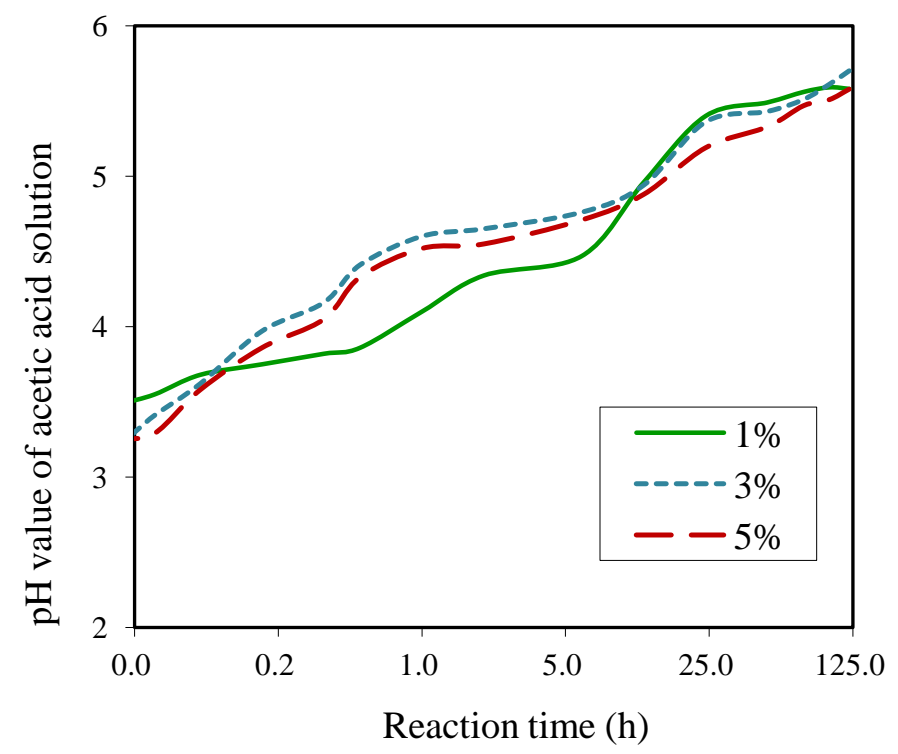

Fig. $2 \mathrm{pH}$ value of the solution varying with the soaking time

\subsubsection{Effects of mechanical rubbing}

Half of the RCA samples immersed in acetic acid for one day were further treated by mechanical rubbing through strong vibration. The purpose of this treatment is to remove the weakened cement mortar from the RCA samples. Fig.3 compares the water absorption of the RCA samples before and after mechanical rubbing. As shown in this figure, mechanical rubbing reduces the water absorptions of the RCA samples by $9.3 \%, 27.9 \%$ and $38.7 \%$ for RCA samples 
treated by $1 \%, 3 \%$ and $5 \%$ acetic acid, respectively. Clearly, this reduction in water absorption is caused by the removal of weakened porous cement mortar from the RCA samples, which is responsible for the high water absorption of the RCA. Fig. 3 suggests that more reduction in water absorption can be achieved by treating the RCA using the acetic acid with higher concentration. This is not surprising since higher concentration of the acid allows for more reaction between the cement mortar and the acid, weakening more mortar or even the NA in the RCA. In this way, more mortar can be removed by mechanical rubbing. This can be verified by measuring the adhered mortar loss of the RCA. Here, the percent of adhered mortar loss $W_{a}$ was defined as [14]:

$$
W_{a}=\frac{M_{1}-M_{2}}{M_{1}} \times 100 \%
$$

where $W_{a}$ is the adhered mortar loss in percent, $M_{1}$ is the dry mass of RCA, and $M_{2}$ is the dry mass of the treated RCA.

Fig.4 clearly shows that more mortar was removed after treatment by the acid with higher concentration. The amount of removed mortar is in proportion to the concentration of the acetic acid, confirming that the loss of the porous mortar is responsible of the absorption reduction observed in Fig.3. 


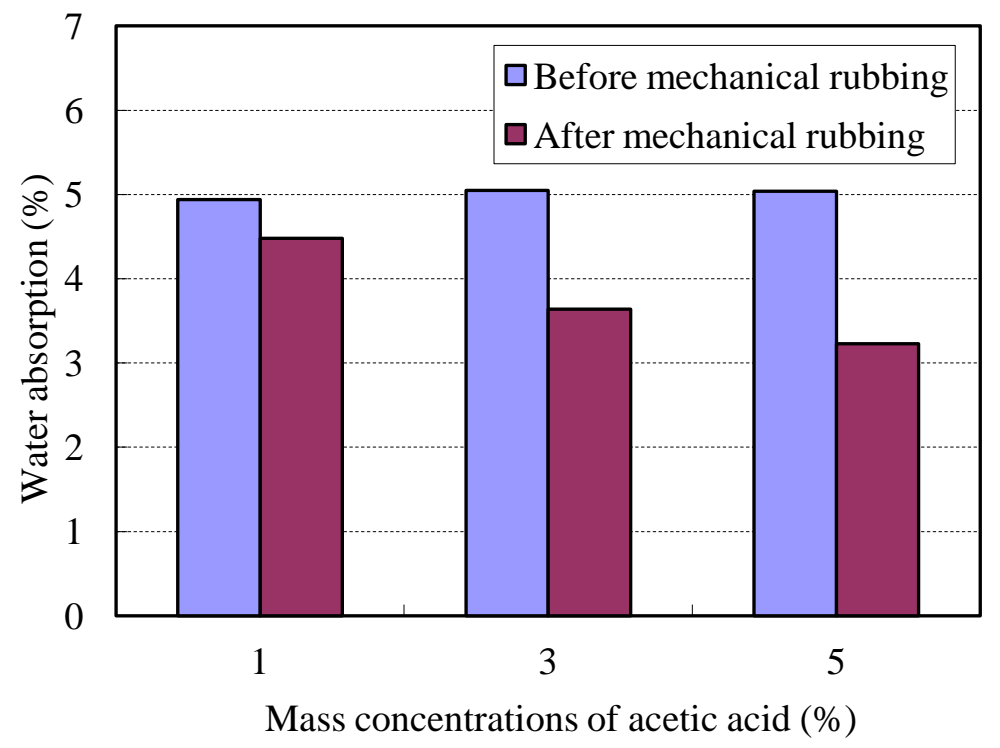

Fig. 3 Water absorption of the acetic acid treated RCA before and after mechanical rubbing

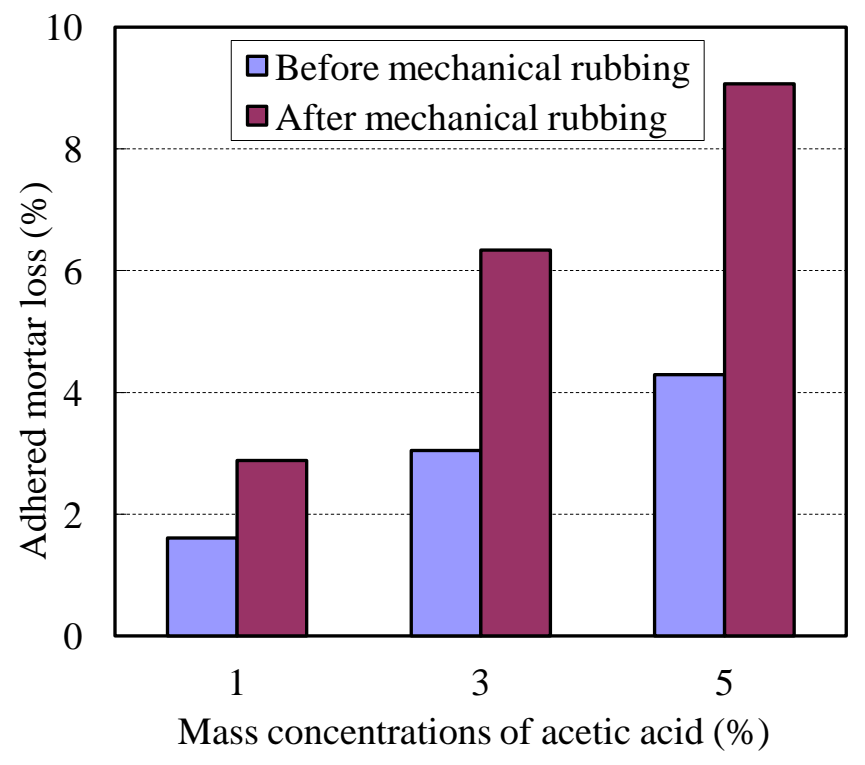

Fig. 4 Adhered mortar loss of the RCA before and after mechanical rubbing

\subsubsection{Surface microstructure of the RCA}

SEM images of the surfaces of the untreated and treated RCA samples are shown in Fig. 5.

The surface of the untreated RCA sample is covered by loosely bonded cement mortar and 
considerably amount of porous fine particles (Fig. 5a), which were created by a crushing process. Similar result was observed by Ismail and Ramli [14]. Fig. 5(b-c) shows the surfaces of RCA samples treated with $1 \%$ and $3 \%$ acetic acids. It can be clearly seen that those loose particles present in the untreated RCA were removed by the acetic acid treatment. This is in agreement with the results on water absorption, apparent density (Table 4), and mortar loss (Fig. 4). Fig. 5d shows the surface of the RCA treated with 5\% acetic acid. Similar to Fig. 5(b-c), loose particles disappeared. However, new pores on the surface of the RCA have been created by the acid treatment, suggesting that damage can be induced to RCA by $5 \%$ acid.

Microstructure of the surfaces of the acid treated RCA samples shown in Figs. 5(b-d) after mechanical rubbing are shown in Fig.5 (e-g). It can be seen that mechanical rubbing made the surfaces of these RCA samples denser and smoother, which is in agreement with findings shown in Figs. (3-4). 

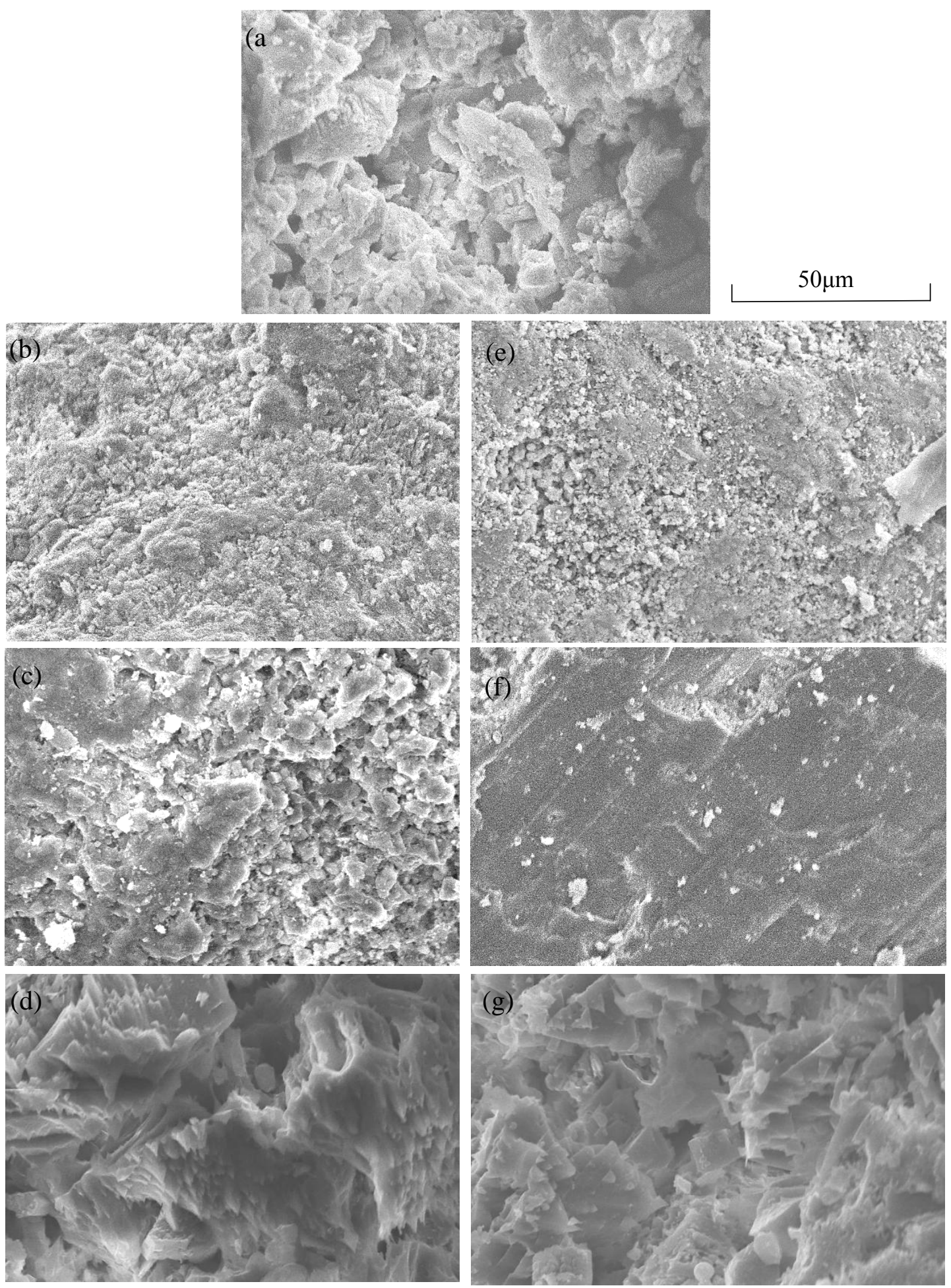

Fig. 5 SEM images of the untreated and treated RCA $(\times 1000)$ : (a) untreated; (b) treated with 1\% acetic acid; (c) treated with $3 \%$ acetic acid; (d) treated with 5\% acetic acid; (e) treated with $1 \%$ acetic acid and mechanical rubbing; (f) treated with $3 \%$ acetic acid and mechanical rubbing; (g) treated with $5 \%$ acetic acid and mechanical rubbing 


\subsubsection{XRD analysis of the RCAs}

Possible chemical reactions between the acetic acid and the RCA are shown in Eqs. (1-3). XRD analysis was carried out to verify whether these reaction occurred and which substances were removed from the RCA by the acetic acid solution. XRD spectra of the untreated RCA sample and the one treated with $3 \%$ acid were obtained and shown in Fig.6. Fig. 6(a) shows that major chemical compositions on the surface of the untreated RCA sample are calcium carbonate, silicon dioxide, and C-S-H. Calcium hydroxide is not shown in Fig.6 (a) because the XRD spectrum was taken on the surface of the RCA, where calcium hydroxide was very likely carbonated by air. After treating with $3 \%$ acetic acid, the contents of calcium carbonate and C-S-H were greatly reduced, as shown in Fig. 6(b), because of the chemical reactions shown in Eqs. (2) and (3). No calcium acetate was found on the XRD spectrum of the $3 \%$ acetic treated RCA sample, as shown in Fig. 6(b) since calcium acetate is dissolvable in water.

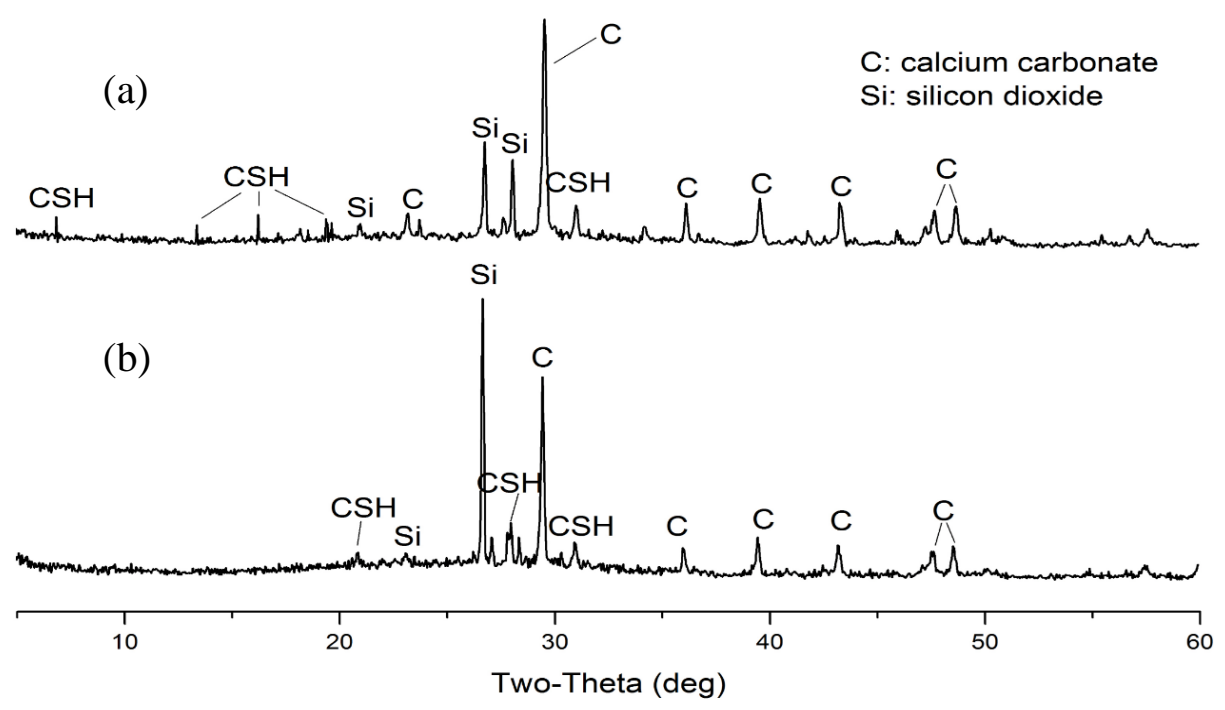

Fig.6. X-ray diffraction (XRD) patterns of the surface of the untreated and treated RCA. 


\subsection{Characterization of the RCA samples and concrete with the RCA}

\subsubsection{Compressive strength of the concrete with the RCA}

The bulk density, slump and compressive strength of the concrete made with the treated and untreated RCAs are shown in Figs. 7, 8, and 9. In these figures, the percentage number shown on the horizontal axis refers to the concentration of the acid used to treat the RCA. The $0 \%$ group is used as reference in which the RCA was not treated. "M", "W", "WM", and "NA" have the same meanings as discussed in Section 2.3.

A few trends can be observed from Fig.7. First, all concretes made with the treated RCA have higher bulk densities than the one with the untreated RCA. This is because some light-weighted cement mortar in the RCA was removed by the treatment. Second, bulk density of the concrete increases with the concentration of the acid because more loose material was removed by the acid, as shown in Fig. 4. The only exception is when 5\% acetic acid was used to treat the RCA without mechanical rubbing. The concrete made with this aggregate has the lowest bulk density among all concretes with the treated RCA, indicating this concrete has a more porous structure. This may be caused by excessive dissolving of hydration products and/or even some NAs in the RCA by the 5\% acetic acid, as shown in Fig. 5(d). Third, mechanical rubbing can further increase the bulk density of the concrete since more loose material was removed from the RCA as shown in Figs.4 and 5. Fig. 7 also shows that washing the RCA after soaking in the acid can slightly reduce the bulk density of the concrete. This is because water washing dissolved and removed some reaction products in the RCA, leaving more pores on the surface of the RCA. 
After removing this surface using mechanical rubbing, the bulk density of the produced concrete was even higher than the one with the RCA treated by mechanical rubbing but without water washing, as shown in Fig. 7.

Fig. 8 shows the slumps of concrete made with different RCAs. It can be seen that the slump of concrete made with treated RCA is slightly higher than the control group. This is mainly because the treated RCAs have lower water absorption. Except $1 \% \mathrm{M}$, mechanical rubbing can further enhance the workability of the concrete because more loose and porous mortar are removed. $1 \%$ acetic acid is weaker than other two concentrations, and therefore, has limited potential to loosen the residual mortar on the RCA as indicated by Figs. 3 and 4. As a result, mechanical rubbing shows negligible effect on the slump of the concrete sample, as shown in Fig.8.

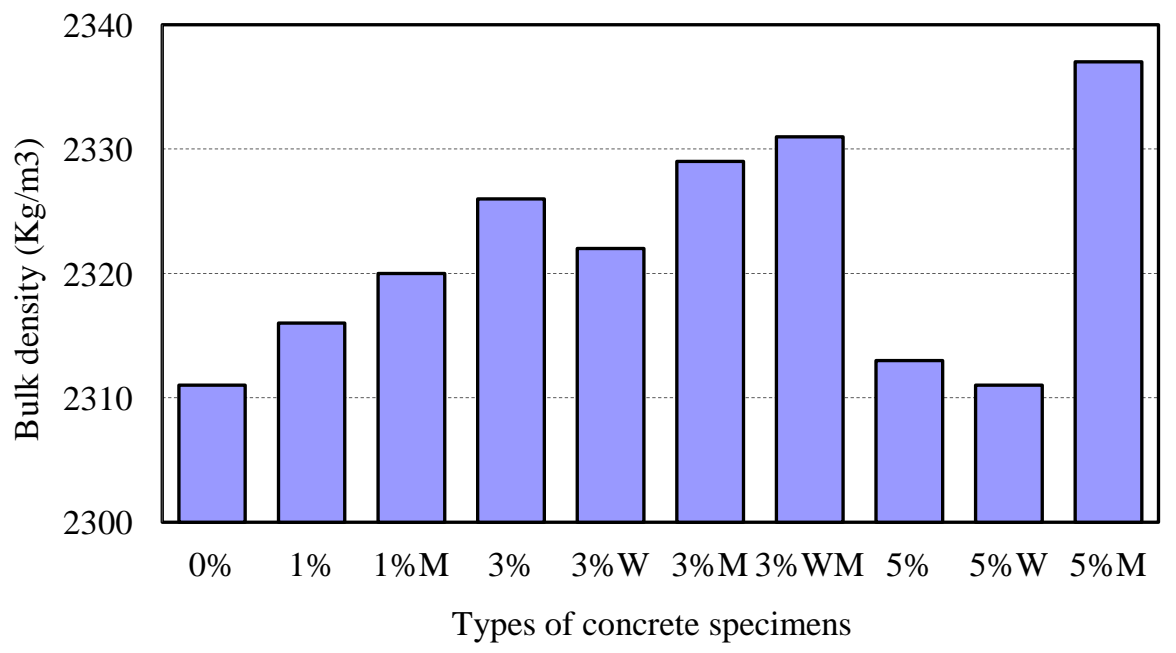

Fig.7 Bulk density of concrete with untreated and the treated RCA 


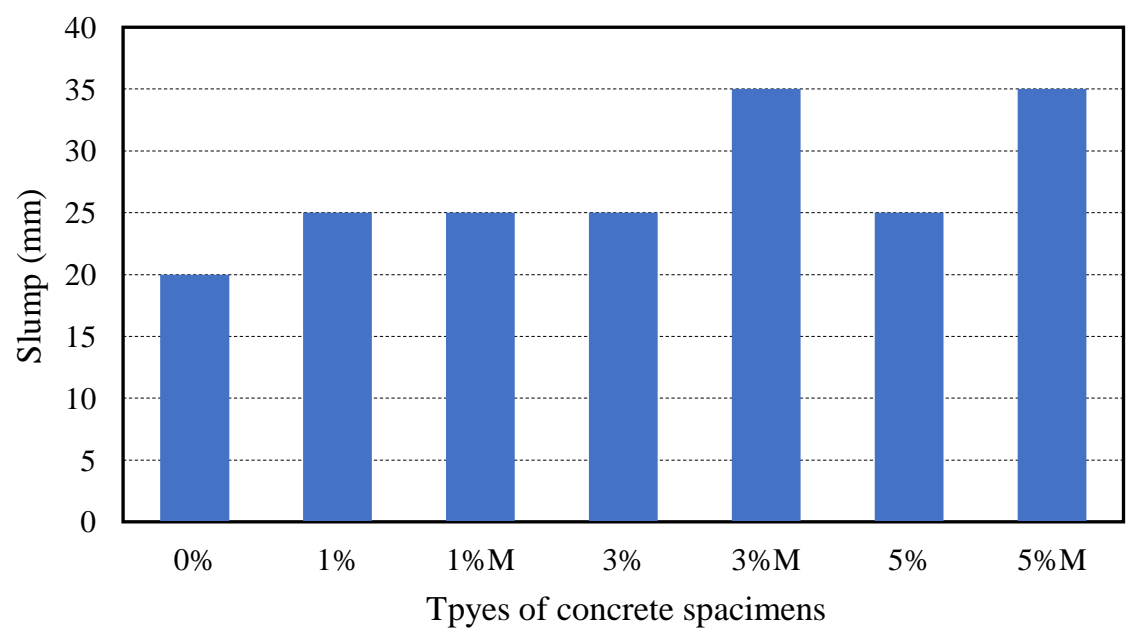

Fig. 8 Slump test results of concrete made with treated and untreated RCA

The compressive strengths of the concretes with the treated RCAs are presented and compared with the control group made with the untreated RCA (0\% group in Fig. 9), as well as the one made with natural aggregate (NA group in Fig. 10) in Figs. 9 and 10. These two figures show that all concrete samples made with the RCA treated with $1 \%$ and $3 \%$ acetic acid have higher compressive strength than the reference group. Over $25 \%$ increase in the compressive strength of the concrete at 28 days has been achieved by the proposed RCA treating method, confirming its effectiveness in improving the quality of the RCA. It can be seen that more increase in the compressive strength of the concrete can be achieved by using $3 \%$ acetic acid to treat the RCA because more loose mortar can be removed, as indicated by Figs. 4 and 5. The effectiveness of the mechanical rubbing is also confirmed by Figs 9 and 10, which show that further strength increase has been obtained after mechanical rubbing.

The effect of the residual acetic acid on the produced concrete can be observed from Figs. 9 and 10 too. Compared with the concrete with unwashed RCA, concrete with washed RCA has 
slightly higher strength at ages of 3 and 7 days. This small difference in early strength is caused by the residual acetic acid in the RCA, which can retard the hydration of cement. At age of 28 days, this retarding effect diminished, as shown in Figs 9 and 10.

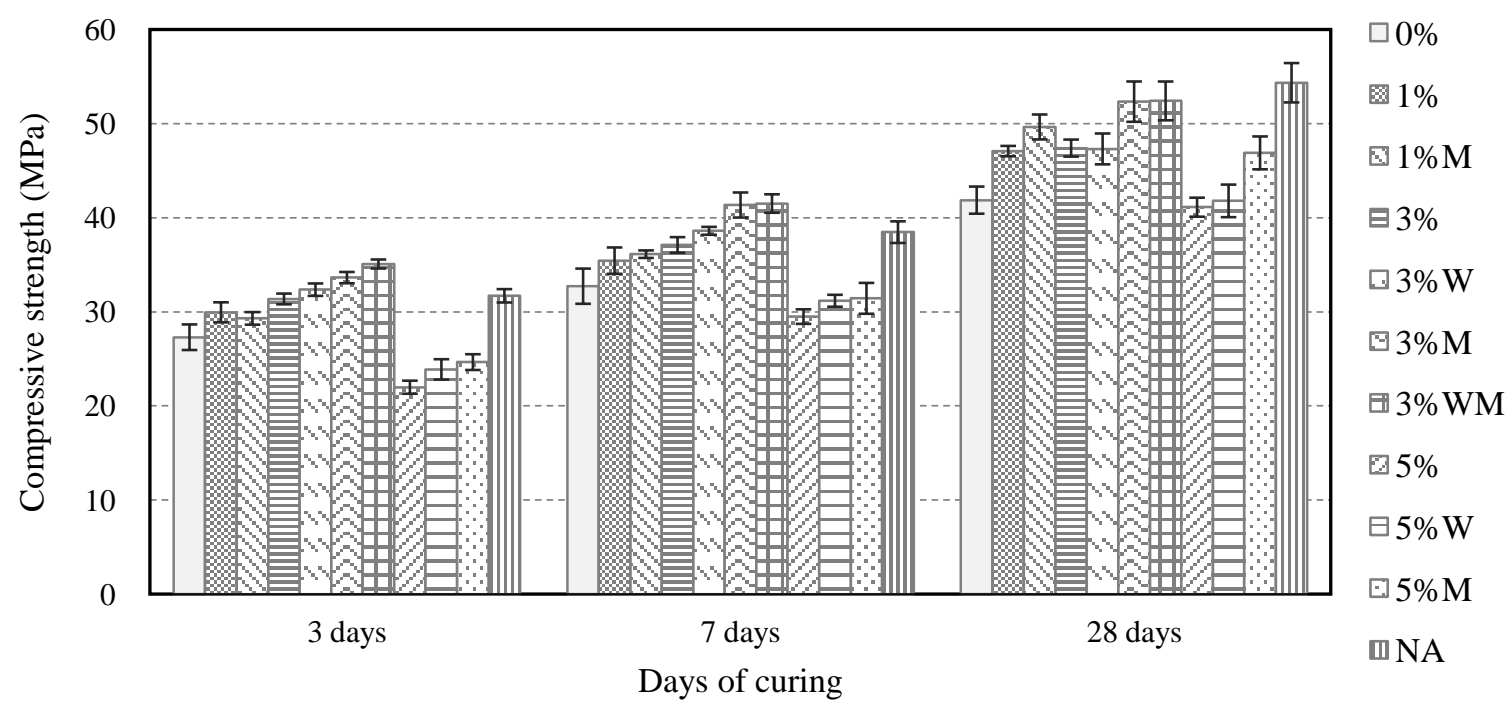

Fig. 9 Compressive strength of concrete with untreated and treated RCAs

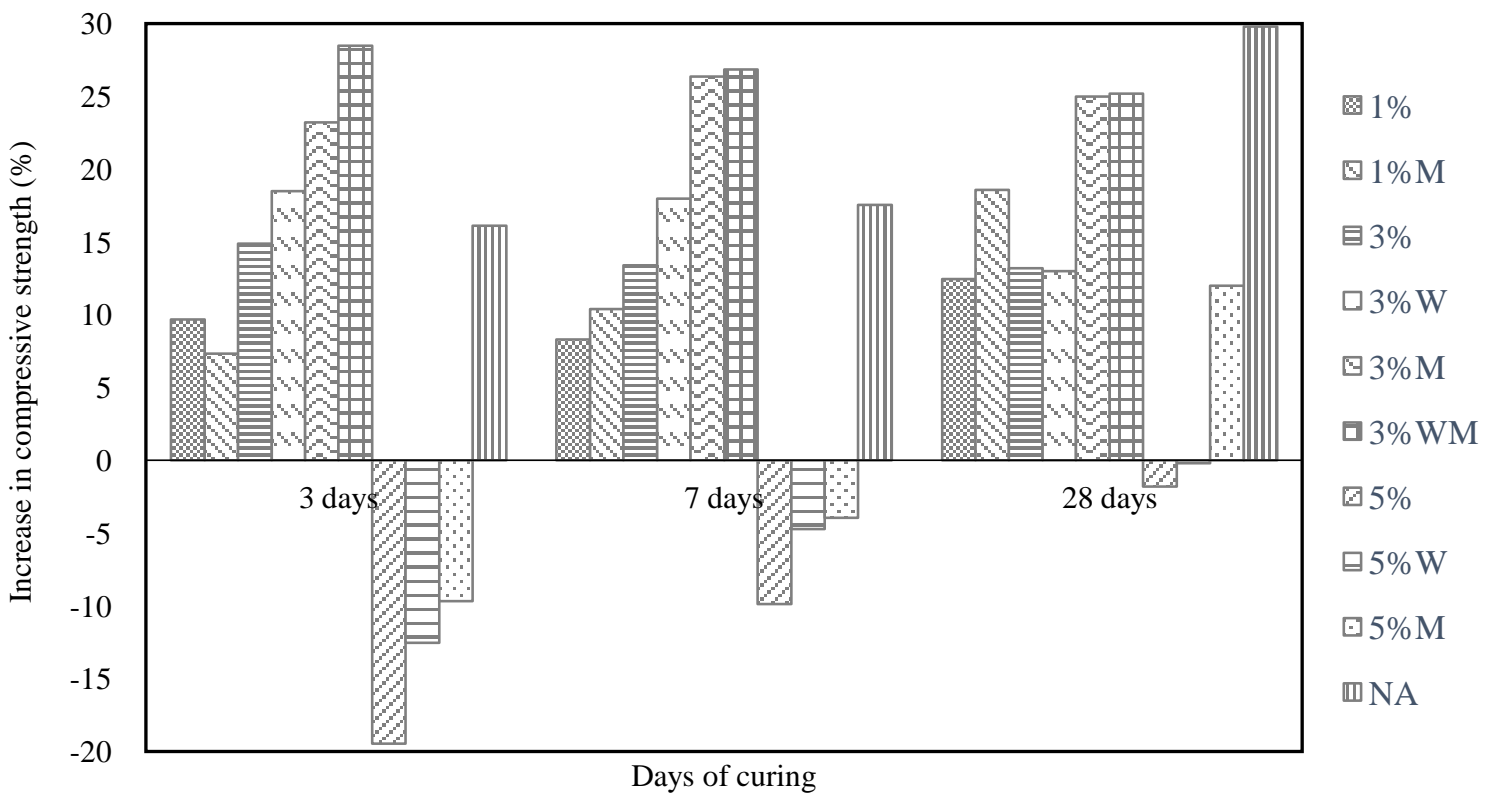

Fig. 10 Compressive strength improvement of concrete with treated RCAs 
However, when $5 \%$ acetic acid was used, the retarding effect of the residual acetic acid became much more pronounced as shown in Fig. 9. Fig. 10 shows that the compressive strength of the concrete was reduced by $19.5 \%$ and $9.9 \%$ at ages of 3 and 7 days, respectively. Similar to the case of using $1 \%$ and $3 \%$ acetic acid solution, the retarding effect of the residual acetic acid appears much smaller at age of 28 days. This retarding effect is not the only reason responsible for the strength reduction in concrete made with the RCA treated with 5\% acetic acid. Another possible reason is the potential damage induced to the RCA by the $5 \%$ acetic acid, which is strong enough not only attack the cement mortar, but also the NA in the RCA, as shown in Fig. $5(d)$.

Compared with the concrete sample made with natural aggregate (NA group), the concrete sample made without treatment has lower strength as expected. However, treating the RCA using the proposed method clearly improve the compressive strength of concrete. Figs. 9 and 10 show that the strength of $3 \% \mathrm{M}$ and $3 \% \mathrm{WM}$ groups at 28 days is only $3.5 \%$ lower than the one with natural aggregate, confirming the effectiveness of the present method on improving the properties of RGAs.

\subsubsection{Microstructures of the concretes made with the RCA}

SEM images of microstructures of the concrete samples made with the untreated and treated RCAs are shown in Fig. 11. Fig. 11(a) clearly shows that there exists a loose zone between the old and the new mortars, which is responsible for the low strength of the concrete with the untreated RCA as shown in Fig.8. This is in agreement with the observation of the microstructure 
of the untreated RCA, as shown in Fig. 5(a). Fig. 11(b) shows that this loose zone was improved by treating the RCA with $1 \%$ acetic acid since some of the loose particles on the surface of the RCA were removed, as shown in Fig. 5(b). Mechanically rubbing the acid treated RCA can further improve this loose zone because more loose materials were removed as indicated in Fig. 5(e). Fig. 11(c) shows that this loose zone was almost completely eliminated in the concrete. Similar trend can be observed for the concrete made with the RCA treated by $3 \%$ acetic acid. It can be seen that the loose zone in this concrete is improved in comparison with that in the concrete made with $1 \%$ acetic acid treated RCA. This is anticipated as $3 \%$ acetic acid removed more loose particles from the RCA, as shown in Figs.3, 4, and 7. As a result, the compressive strength of this concrete is slightly higher than that of the concrete made with $1 \%$ acetic acid treated RCA, as shown in Fig. 8. Fig. 11(f) shows the microstructures of the concrete made with RCA treated by $5 \%$ acetic acid. It can be seen a much smaller loose zone present in this sample because of high reactivity between the acetic acid and the RCA. Multiple porous spots can be identified from Figs. 11(f) and (g), which are residuals of minerals dissolved by the 5\% acetic acid. These porous spots can weaken the compressive strength of the concrete. The overall dense microstructure of the concrete using the RCA treated with 5\% acetic acid suggests that the retarding effect of acetic acid is the major reason for the lower strength of the concrete shown in Fig. 9. 


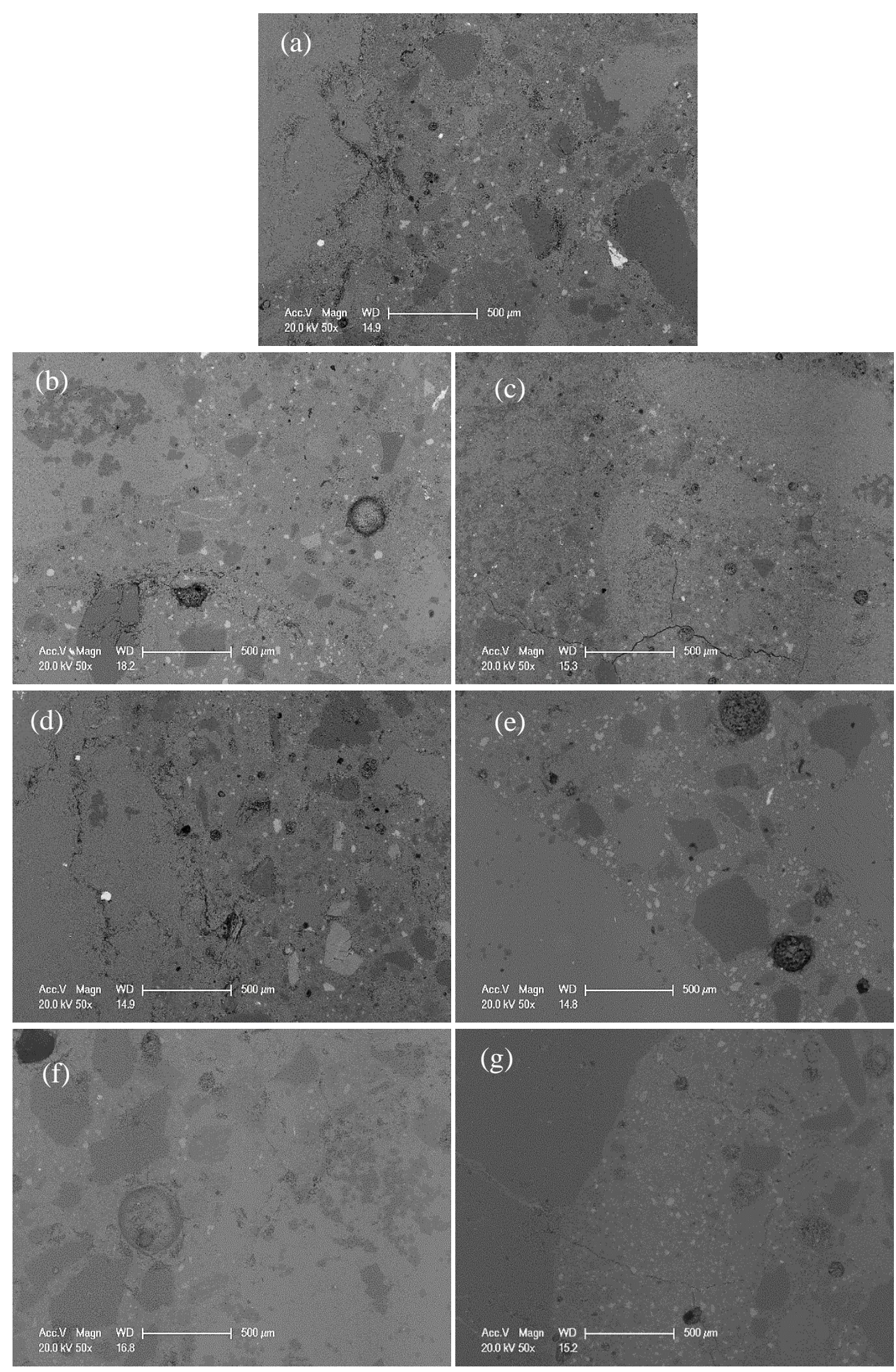

Fig.11 Microstructure of the concrete using the RCA: (a) without treatment; (b) treated with $1 \%$ acetic acid; (c) treated with $1 \%$ acetic acid and mechanical rubbing; (d) treated with $3 \%$ acetic acid; (e) treated with 3\% acetic acid and mechanical rubbing; (f) treated with 5\% acetic acid; (g) treated with $5 \%$ acetic acid and mechanical rubbing. 


\subsection{Chemical compositions in the waste solution of the treatment}

DEM and EDS analyses were carried out on the residual powders distilled from the waste solution of the treatment. As shown in Fig. 12, major elements existing in waste solution are calcium $(\mathrm{Ca})$, oxygen $(\mathrm{O})$ and carbon $(\mathrm{C})$, which are from reaction products shown in Eqs. (1-3). In addition, small amount of aluminum (Al), magnesium $(\mathrm{Mg})$ and silicon $(\mathrm{Si})$ are present, which are from the dissolved cement mortar.
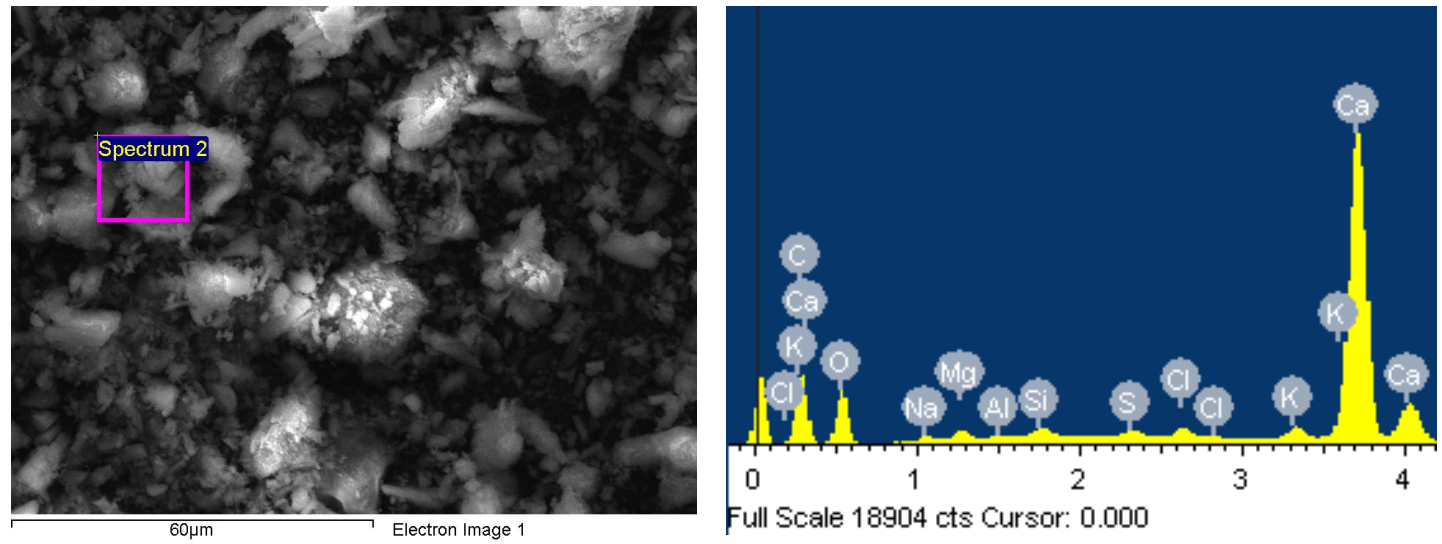

Fig. 12 EDS analysis of the residual powders in waste solution

XRD spectrum (Fig.13) was obtained for the waste solution after precipitating metal elements. It can be clearly seen that the main chemical composition in the waste solution is the acetate hydrate $\left(\mathrm{Ca}(\mathrm{Ac})_{2} \cdot \mathrm{H}_{2} \mathrm{O}\right)$. 


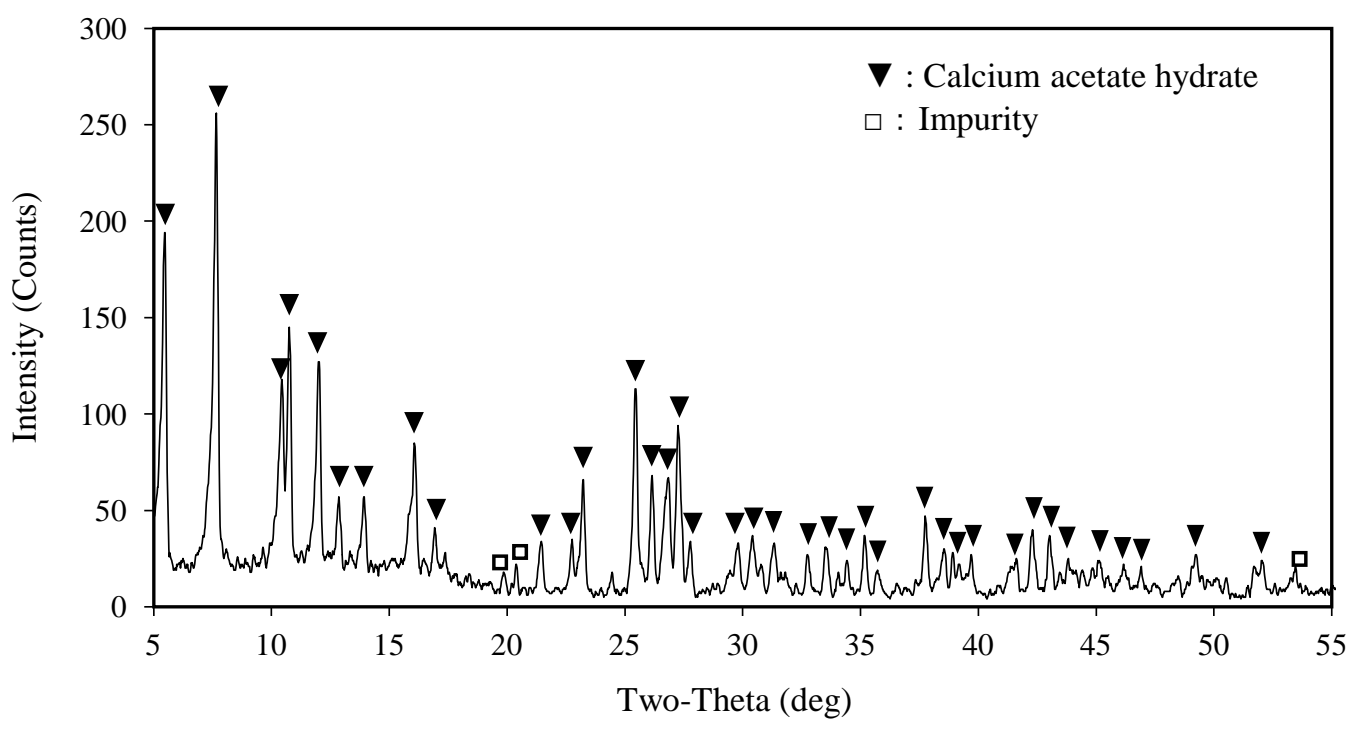

Fig.13 X-ray diffraction (XRD) patterns of waste solution

\subsection{Strength improvement induced by using the waste solution as an admixture}

Table 5 shows that the compressive strength of the concrete samples with and without using the waste solution as the chemical admixture. In this table, Group "W" is the control group in which no waste solution was added. Group "S1(3,5)\%" refers to the concrete sample made with the waste solution generated after treating the RCA with $1(3,5) \%$ acetic acid. The concentration of calcium ions in these solutions were measured, as shown in Table 6. It can be seen that the strengths of all concrete samples were improved by the waste solution of the treatment. Among them, S3\% group exhibits the highest improvement in strength. At age of 28 days, this group of concrete has a strength exceeding the control one by $13.8 \%$. This indicates that calcium acetate in waste solution can improve the compressive strength of concrete. The mechanism responsible for this strength improvement is under study. But one possible mechanism is the calcium ions in the solution, which can react quickly with cement to produce $\mathrm{C}-\mathrm{S}-\mathrm{H}$ or $\mathrm{CH}$, leading to denser 
microstructure. In this way, waste solution of the treatment can be directly added into concrete to improve the strength of the concrete.

Table 5 Compressive strength of concrete using the waste solution as an admixture

\begin{tabular}{llllllllll}
\hline & \multicolumn{3}{l}{3 days age } & \multicolumn{3}{c}{7 days age } & \multicolumn{3}{c}{ 28 days age } \\
\cline { 2 - 10 } Group & $\begin{array}{l}f_{\text {cu, } m} \\
(\mathrm{MPa})\end{array}$ & STD & $\begin{array}{c}\text { Increase } \\
(\%)\end{array}$ & $\begin{array}{l}f_{\text {cu, } m} \\
(\mathrm{MPa})\end{array}$ & STD & $\begin{array}{l}\text { Increase } \\
(\%)\end{array}$ & $\begin{array}{l}f_{\text {cu, } m} \\
(\mathrm{MPa})\end{array}$ & STD & $\begin{array}{l}\text { Increase } \\
(\%)\end{array}$ \\
\hline $\mathrm{W}$ & 31.35 & 1.15 & - & 39.09 & 1.10 & - & 46.93 & 2.08 & - \\
$\mathrm{S} 1 \%$ & 30.13 & 1.07 & -3.9 & 39.24 & 0.83 & 0.4 & 49.06 & 1.14 & 4.5 \\
$\mathrm{~S} 3 \%$ & 33.09 & 1.81 & 5.6 & 43.96 & 0.40 & 12.5 & 53.40 & 1.24 & 13.8 \\
$\mathrm{~S} 5 \%$ & 31.65 & 0.28 & 1.0 & 41.90 & 0.36 & 7.2 & 49.56 & 2.11 & 5.6 \\
\hline
\end{tabular}

Table 6 Calcium ion concentration in waste solution

\begin{tabular}{llll}
\hline Series no. & S1\% & S3\% & S5\% \\
\hline Concentration $(\mathrm{g} / \mathrm{L})$ & 2.03 & 7.27 & 12.80 \\
\hline
\end{tabular}

\section{Conclusions}

An environmentally friendly method has been proposed and successfully demonstrated to improve the quality of the RCA using acetic acid to remove loose particles from the RCA. Compared with existing chemical processing method using strong acids, the new method is safer and cleaner in that: 1) no strong acid is used;2) no detrimental chemical is introduced into the RCA, and 3) no hazardous waste material is produced. Experimental studies carried out in this study suggest that:

(1) Soaking in the acetic acid solution can effectively remove cement mortar on the surface of the RCA. The quality of the RCA such as water absorption, apparent density, can be improved by this treatment. In ambient environment, 24h soaking in the acetic solution 
appears sufficient. The compressive strength of the concrete made with the RCA can be significantly improved by the proposed treating method.

(2) The residual acetic acid has very little effect on the strength development of the concrete when low concentration of the acetic acid is used. Significant retarding effect was observed when $5 \%$ acetic acid is used. Therefore, acid concentration no more than $3 \%$ is recommended for RCA treating to avoid the retarding effect.

(3) Mechanical rubbing after acid soaking can further remove loose particles from RCAs and enhance the qualities of RCAs and the concrete with RCAs.

(4) The waste solution of this treatment can be used as a chemical admixture in concrete to significantly improve the strength of the concrete. In this way, no waste is produced by the proposed method.

It should be pointed out this study is just the proof-of-concept of the new method. More improvement on RCA can be achieved by optimizing the acetic soaking conditions and mechanical rubbing, which will be examined in future study.

\section{Acknowledgements}

This study was partially supported by the National Science Foundation - United States CMMI - 1000580. Any Opinions, findings and conclusions or recommendations expressed in this material are those of the author(s) and do not necessarily reflect those of the National Science Foundation. 


\section{References}

[1].T.R. Sonawane, S.S. Pimplikar, Use of recycled aggregate in concrete, Int J Eng Res Technol 2(2013) 1-9.

[2].M. Behera, S.K. Bhattacharyya, A.K. Minocha, R. Deoliya, S. Maiti, Recycled aggregate from $\mathrm{C} \& \mathrm{D}$ waste $\&$ its use in concrete-A breakthrough toward sustainability in construction sector: A review, Construction and building materials 68 (2014) 501-516.

[3].N.D. Oikonomou, Recycled concrete aggregates, Cem Concr Compos 27 (2005) 315-318.

[4].J.Z. Xiao, W.G. Li, C.S. Poon, Recent studies on mechanical properties of recycled aggregate concrete in China-A review, Technological Sciences 55 (2012) 1463-1480.

[5].R.S. Ravindrajah, , Y.H. Loo, C.T. Tam, Strength evaluation of recycled aggregate concrete by in-situ tests, Materials and Structures 21 (1988) 289-295.

[6].S.M. Levy, P. Helene, Durability of recycled aggregate concrete: a safe way to sustainable development, Cement and Concrete Research 34 (2004) 1975-1980.

[7].A. Domingo-Cabo, C. López-Gayarre, M.A. Serrano-López, P. Serna, J.O. Castano-Tabares, Creep and shrinkage of recycled aggregate concrete, Construction and Building Materials 23 (2009) 2545-2553.

[8].E. Mulder, T.P.R. de Jong, L. Feenstra. Closed Cycle Construction: An integrated process for the separation and reuse of C\&D waste, Waste Management 27 (2007) 1408-1415.

[9].J.A. Larbi, W.M.M. Heijnen, J.P. Brouwer, E. Mulder, Preliminary laboratory investigation of thermally treated recycled concrete aggregate for general use in concrete. In: Woolley JJJMG 
GR, Wainwright PJ, editors. Waste management series. Elsevier; 2000, p. 129-139.

[10]. A. Akbarnezhad, K.C.G. Ong, M.H. Zhang, C.T. Tam, T.W.J. Foo, Microwave-assisted beneficiation of recycled concrete aggregates, Construction and Building Materials 25 (2011) 3469-3479.

[11]. H. Shima, H. Tateyashiki, R. Matsuhashi, Y. Yoshida, An advanced concrete recycling technology and its applicability assessment through input-output analysis, J Adv Concr Technol 3 (2005) 53-67.

[12]. A. Katz, Treatments for the improvement of recycled aggregate, J Mater Civ Eng 16 (2004) 597-603.

[13]. V.W.Y. Tam, C.M. Tam, K.N. Le, Removal of cement mortar remains from recycled aggregate using pre-soaking approaches, Resour Conserv Recycl 50 (2007) 82-101.

[14]. S. Ismail, M., Ramli, Engineering properties of treated recycled concrete aggregate (RCA) for structural applications, Construction and Building Materials 44 (2013) 464-476.

[15]. B.H. Chollar, Y. P. Virmani, Effects of calcium magnesium acetate on reinforced steel concrete, Public Roads 51 (1988) 113-115.

[16]. C. Balachandran, J. Olek, P. Rangaraju, S. Diamond, Role of potassium acetate deicer in accelerating alkali-silica reaction in concrete pavements: Relationship between laboratory and field studies, Transportation Research Record 2240 (2011)70-79.

[17]. G. Klnççeker, C. Meneke, The effect of acetate ions on the corrosion of reinforcing steel in chloride environments, Protection of Metals and Physical Chemistry of Surfaces 51 (2015) 659-666. 
[18]. P. Chen, J. Wang, L. Wang, Y. Xu, X. Qian, H. Ma, Novel production of a useful product, vaterite, by $\mathrm{CO} 2$ sequestration in the waste solution generated during treatment of recycled concrete aggregate. Journal of Cleaner Production 149 (2017) 735-742.

[19]. I. Udrea, C. Capat, E.A. Olaru, R., Isopescu, M., Mihai, C.D. Mateescu, C. Bradu, Vaterite Synthesis via Gas-Liquid Route under Controlled pH Conditions, Industrial \& Engineering Chemistry Research 51 (2012) 8185-8193.

[20]. D.B. Trushina, T.V. Bukreeva, M.V. Kovalchuk, M.N. Antipina, $\mathrm{CaCO}_{3}$ vaterite microparticles for biomedical and personal care applications, Materials Science and Engineering: C 45 (2014) 644-658.

[21]. J.D. Rodriguez-Blanco, S. Shaw, L.G. Benning, The kinetics and mechanisms of amorphous calcium carbonate (ACC) crystallization to calcite, via vaterite, Nanoscale 3 (2011) 265.

[22]. I. Chen, T.P. Lee, J. Patterson, Concrete compositions and methods. US Patent application US2013/0167756A1, 2013. 